\title{
Fluorescence Microscopy of Corrole-Single Silver Nanowire Hybrid Nanostructures
}

\author{
N. Czechowski ${ }^{a}$, M. Olejnik ${ }^{a}$, A. Nowak-Król ${ }^{b}$, D. PiątKowski ${ }^{a}, W \cdot \operatorname{Heiss}^{c}$, \\ D.T. GRYKO ${ }^{b, *}$ AND S. MACKOWSKI ${ }^{a}$ \\ ${ }^{a}$ Institute of Physics, Nicolaus Copernicus University, Grudziądzka 5, 87-100 Toruń, Poland \\ ${ }^{b}$ Institute of Organic Chemistry, Polish Academy of Sciences, Warsaw, Poland \\ ${ }^{c}$ Institute of Solid State Physics, Johanes Kepler University, A-4040 Linz, Austria
}

We report on confocal microscopy imaging of hybrid nanostructures composed of silver nanowires and corroles. Both nanomaterials were separated by a $30 \mathrm{~nm}$ thick $\mathrm{SiO}_{2}$ spacer in order to inhibit fluorescence quenching. The results show that for such a hybrid nanostructure the average enhancement of the fluorescence intensity reached 2.5. Importantly, the coupling to plasmon excitations in metallic nanowires leaves no effect on the fluorescence spectrum of the organic molecules.

PACS: 33.50.-j, 78.67.Uh, 73.20.Mf, 81.07.Pr, 81.07.-b

\section{Introduction}

Optical fluorescence microscopy [1] is a universal method for investigating among others photophysical processes of molecules and their assemblies, interactions at the nanoscale such as energy transfer or plasmon enhanced phenomena, as well as cellular dynamics. High-sensitivity detectors such as single photon counting avalanche photodiode modules or multiplying CCD matrices combined with sub-wavelength spatial resolution allow for studying fluorescence properties of individual nanostructures [2]. The spatial resolution of a microscopy system can be improved by using liquidor solid-immersion techniques $[1,3-5]$ or via exploiting evanescent field excitation, like in the near-field optical microscopy [6] or total internal reflection microscopy [1].

One of the physical processes widely studied using fluorescence spectroscopy and microscopy is plasmon-induced enhancement of the optical properties of $\mathrm{flu}$ orophores placed in the vicinity of metallic surfaces/ nanoparticles [7]. It has been shown for many systems that under properly designed geometry the emission and absorption of a fluorophore can be significantly increased due to local electromagnetic field induced by plasmon in a metallic nanoparticle [7-9]. In addition to simple fluorophores, recently also more complex structures such as pigment protein complexes that participate in photosynthesis have been shown to improve their function upon coupling with metallic nanoparticles [10-13]. The impact of plasmon interactions on the optical properties of a fluorophore depends on several parameters, including the spectral properties of the building blocks of a hybrid nanostructure as well as the separation between them. For instance, when the separation is too small, typically below $5 \mathrm{~nm}$, the dominant process is a quench-

* corresponding author; e-mail: dtgryko@icho.edu.pl ing of fluorescence due to nonradiative energy transfer to the metallic nanoparticle.

In this work we apply confocal fluorescence microscopy to investigate the interaction between plasmons localized in silver nanowires and an organic molecule, a 5,10,15-tris(pentafluorophenyl)corrole. The silver nanowires are special in the family of metallic nanoparticles as they are characterized with huge aspect ratio: while diameters of the nanowires are below $100 \mathrm{~nm}$, their length is in the range of tens of $\mu \mathrm{m}$. Therefore, it is possible to image them using standard microscopy. In order to control the separation between the nanowires and corroles we use a $30 \mathrm{~nm}$ thick $\mathrm{SiO}_{2}$ spacer. The corroles on the other hand belong to the tetrapyrrolic family of organic molecules, resembling porphyrins and corrins. Since developing efficient ways of synthesizing corroles [14] these molecules have gained a lot of interest, mainly from the point of view of photodynamic antitumor therapy, biosensing, and catalysis. The results of optical fluorescence microscopy obtained for a hybrid nanostructure consisting of a single silver nanowire and dye molecules deposited at a fixed distance show the enhancement of emission intensity due to coupling to plasmon excitations. As corroles are becoming possible building block of complex molecular dyads suitable for light-harvesting [15] this observation can be important for devising novel nanostructures for artificial photosynthesis.

\section{Materials and methods}

$5,10,15$-tris(pentafluorophenyl)corrole is one of the most studied corrols, it has been synthesized using published procedure [16].

We synthesized silver nanowires using polyol process in which the ethylene glycol served as the reducing and solvent reagent [17]. This method gives us an opportunity to carry out reaction in the soft condition with temperatures $<200^{\circ} \mathrm{C}$ and atmospheric pressure. Silver nitrate is 
reduced by ethylene glycol in the presence of poly (vinylpyrrolidone) (PVP). PVP acts as a stabilizing agent creates a protective coating onto the silver surface thus preventing from aggregation of silver nanowires. In typical synthesis, $40 \mu \mathrm{l}$ of $\mathrm{CuCl}_{2}$ solution ( $4 \mathrm{mM}$ in EG) was added to $5 \mathrm{~mL}$ of ethylene glycol in glass vial, heated at $150^{\circ} \mathrm{C}$ with stirring. After $15 \mathrm{~min}, 1.5 \mathrm{~mL}$ of PVP solution (114 mM in EG) and $1.5 \mathrm{~mL} \mathrm{AgNO}_{3}$ solution (94 $\mathrm{mM}$ in EG) was then simultaneously added dropwise using a syringe pump. The reaction mixture was continued with heating for one hour. The product was purified by centrifugation at $2000 \mathrm{rpm}$ for $20 \mathrm{~min}$ and redispersed in pure water.

In order to characterize the molecule, we dissolve it in HPLC grade acetone to the concentration of $0.05 \mathrm{mg} / \mathrm{ml}$. The nanowires on the other hand were dissolved in water with concentration adjusted to obtain OD equal to 1 for the absorption maximum. Absorption spectra of the corrole and the nanowires were measured using Perkin Elmer Lambda 2 spectrometer. The solution was placed in a disposable quvette (Eppendorf) and sealed on top to prevent evaporation during the measurement. Similar approach was used to measure fluorescence spectra but the concentration used was $0.01 \mathrm{mg} / \mathrm{ml}$. They were obtained using Fluorolog 3 spectrofluorimeter (JobinYvon) with a xenon lamp as an illumination source.

A schematic drawing of our structure is shown in Fig. 1. Hybrid nanostructures were obtained by depositing nanowires on a clean glass microscope slides, washed in acetone and methanol, and coating them with a silica layer. $50 \mu \mathrm{l}$ of nanowire solution was spin-coated on a glass slide. The concentration of the nanowires was adjusted to the level that upon the spin coating resulted in a layer of well-separated nanowires. The density of the nanowires on a substrate was checked with a scanning electron microscope after coating the layer of nanowires with a thin $2 \mathrm{~nm}$ gold layer. An example of the SEM image is shown in the inset to Fig. 1. Such prepared subtrates were next covered with a $30 \mathrm{~nm}$ thick layer of silica using e-beam evaporation technique. In the final step corroles were deposited in a PMMA matrix.

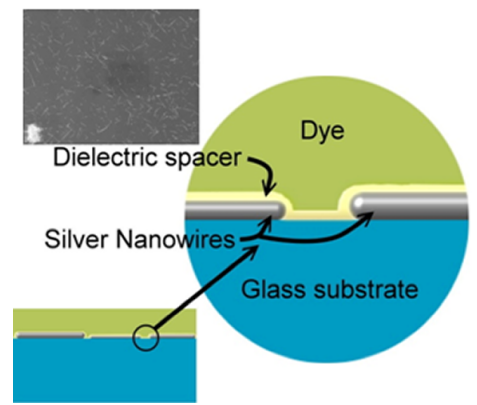

Fig. 1. The schematics of the fabricated hybrid nanostructure. Scale between nanowires diameter and thickness of the dielectric spacer is preserved, the thickness of the dielectric spacer is equal to $30 \mathrm{~nm}$ and the diameters of the nanowires vary from $50 \mathrm{~nm}$ to $200 \mathrm{~nm}$.
Fluorescence properties of the structure were studied using a home-built confocal fluorescence microscope [5] based on a piezoelectric stage (E-501.00, Physik Instrumente) that enabled precise movement of the sample. The accuracy was better than $10 \mathrm{~nm}$, and the range was $100 \mu \mathrm{m}$ in both $X$ and $Y$ directions. By correlating the movement of the piezoelectric stage with the readout of the avalanche photodiode detector (SPCM-AQRH-14, Perkin Elmer) we obtain a fluorescence map of the sample. The excitation was provided by two lasers: $532 \mathrm{~nm}$, at the wavelength which is very weakly absorbed by the corroles, and $405 \mathrm{~nm}$, at the wavelength which is strongly absorbed by the corroles. The first laser enable us to obtain a reflection image when coupled with a narrow laser filter $(532 / 2)$ on the detector. In order to isolate the emission of the corrole molecules and reduce any stray light we use a bandpass filter (HQ 670/10, Chroma). The experiment was carried out in such a way that at first we optically located a silver nanowire utilizing $532 \mathrm{~nm}$ laser and monitoring its reflection and then measuring the $670 \mathrm{~nm}$ fluorescence of the corrole excited with a $405 \mathrm{~nm}$ laser. In addition to fluorescence maps we have also a possibility to collect fluorescence spectra at a given location on the sample. The spectral dispersion is achieved using an Amici prism coupled with a thermoelectrically cooled back-thinned CCD detector (iDus, Andor). For excitation and detection a $50 \times$ microscope objective with numerical aperture 0.5 was used which provided approximately $1.5 \mu \mathrm{m}$ spatial resolution of our confocal microscope.

\section{Experimental results}

In Fig. 2 we show absorption (black curve) and emission (red curve) spectra measured for corrole diluted in acetone. The absorption features three well resolved bands at $586 \mathrm{~nm}, 639 \mathrm{~nm}$, and $437 \mathrm{~nm}$ which correspond to $Q_{x}, Q_{y}$ and Soret band, respectively. The emission excited at $439 \mathrm{~nm}$ shows a maximum at $654 \mathrm{~nm}$ and a weak phonon replica at $717 \mathrm{~nm}$. These results are similar to previously published [18] but exhibit some small vari-

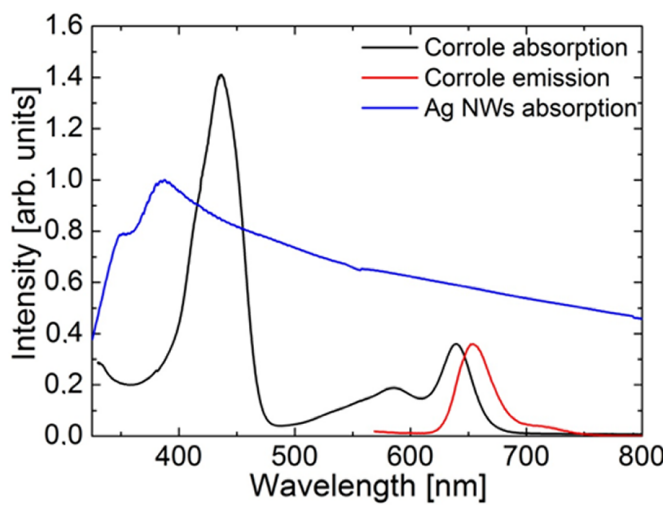

Fig. 2. Absorption (black) and emission (red) of 5,10,15-tris(pentafluorophenyl)corrole dissolved in acetone in room temperature. The blue line represents absorption of the silver nanowires in water solution. 
ation, possibly due to change of the solvent from toluene to acetone. The optical spectra of the corrole are compared in Fig. 2 with the absorption of the silver nanowires in water (blue curve). The maximum absorption in this case is located at $390 \mathrm{~nm}$ with a shoulder at $350 \mathrm{~nm}$. It is important to note that although the absorption peak is very broad, its maximum corresponds well to the Soret band absorption of the corrole molecules (for $437 \mathrm{~nm}$ the nanowires exhibit $85 \%$ of maximum absorption). For the excitation wavelength used in the experiment $(405 \mathrm{~nm})$ the absorption of the corroles and nanowires equals to $41 \%$ and $94 \%$ of their maxima, respectively. At the same time, the corroles absorb almost no light at the wavelength of $532 \mathrm{~nm}$.
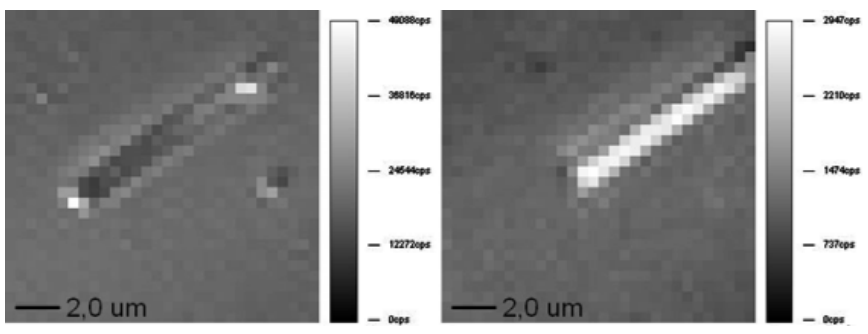

Fig. 3. Maps obtained for the corrole sample with $30 \mathrm{~nm} \mathrm{\textrm {SiO } _ { 2 }}$ spacer. On the left: reflection image obtained by reflecting $532 \mathrm{~nm}$ laser, on the right: fluorescence map of the same region, measured using $405 \mathrm{~nm}$ laser for excitation and monitoring the $670 \pm 5 \mathrm{~nm}$ fluorescence emission.

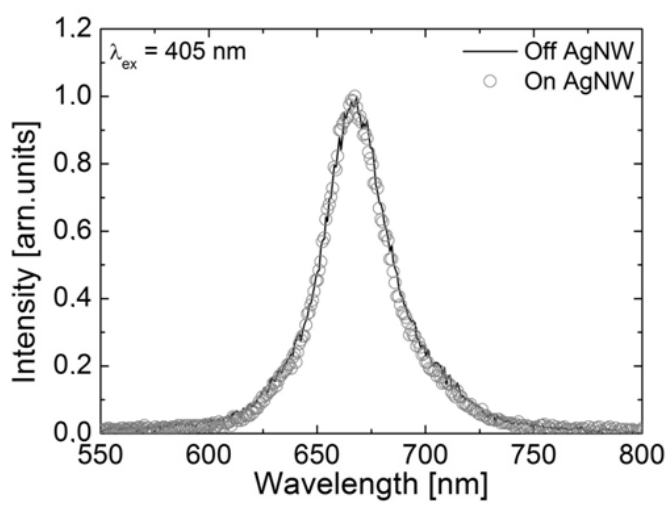

Fig. 4. Fluorescence emission spectra of corroles measured on top of the silver nanowire (gray circles) and off the nanowire (black solid line). The spectra are normalized for better comparison.

The result of microscope imaging of both the nanowires and the corrole fluorescence is shown in Fig. 3. The reflection image of the sample (Fig. 3a) measured for the $532 \mathrm{~nm}$ excitation laser shows clearly the position of a silver nanowire. Subsequent acquisition of the fluorescence image (Fig. 3b) of corroles allows to correlate both images in a straightforward way. The fluorescence image features strong emission for corrole molecules deposited directly on top of the nanowire, while the intensity off the nanowire is considerably lower. In order to demonstrate that the corroles are distributed all over the sample we measured fluroescence spectra both on and off the nanowire. The result is displayed in Fig. 4. For both areas the fluorescence spectrum is virtually identical indicating that, on the one hand, the corroles are indeed spread out on the $\mathrm{SiO}_{2}$ spacer layer, and, on the other hand, that interaction with plasmon excitations in metallic nanowire induced no changes in the shape of the emission.

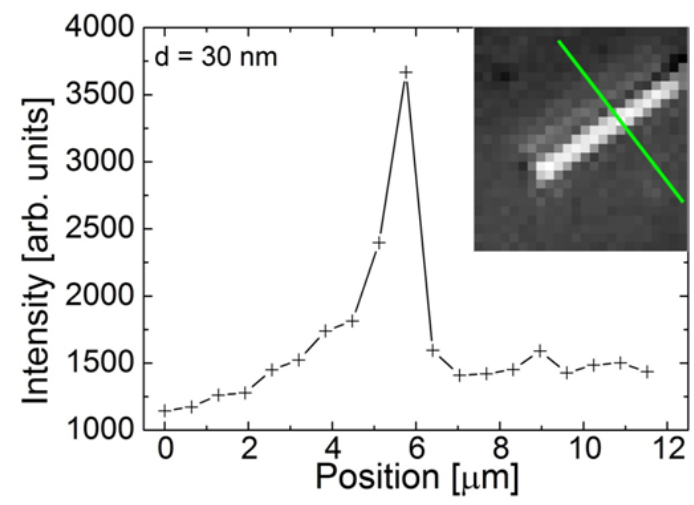

Fig. 5. Cross-section of the fluorescence map in the direction perpendicular to the silver nanowire. In the inset the actual geometry is presented.

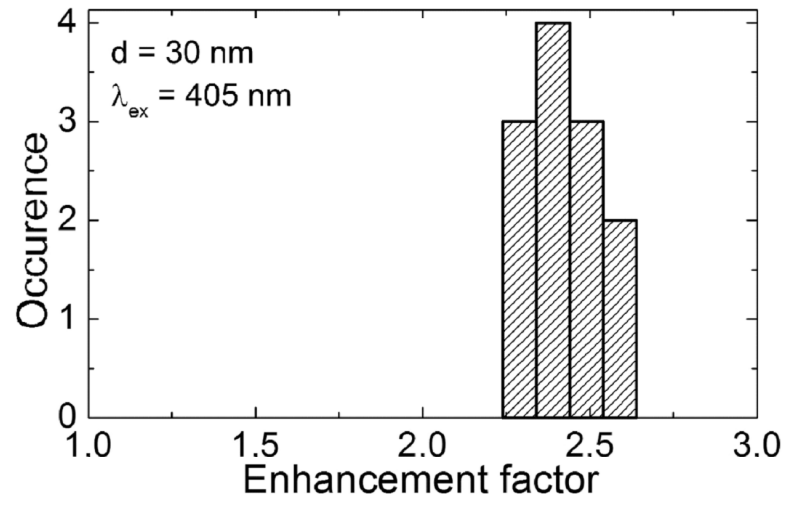

Fig. 6. Histogram of enhancement factors calculated for 12 cross-sections made for the same silver nanowire.

The enhancement factor of the corrole fluorescence for this hybrid nanostructure was estimated by calculating 12 cross-sections of the fluorescence intensity in the direction perpendicular to the nanowire axis. An example of such a cross-section is shown in Fig. 5. While the fluorescence intensity off the nanowire is around $1200 \mathrm{cps}$, it goes over $3600 \mathrm{cps}$ in the middle of the nanowire. We calculate the enhancement factor by dividing the intensity of the fluorescence on the nanowire by the average intensity of the fluorescence off the silver nanowire. The latter is typically equal to $1400 \mathrm{cps}$. For the cross-section 
displayed in Fig. 5 the enhancement factor is 2.54. Statistical analysis of the enhancement factors for all 12 cross-sections is shown in Fig. 6. The average enhancement factor for corrole fluorescence in the investigated sample was calculated at $2.4 \pm 0.1$. We note that such a method of estimating the value of fluorescence enhancement neglects the effect of a dark count rate of the detector. However, for the single photon detectors used in the experiment the background was found to be less than $100 \mathrm{cps}$ in our experimental configuration. The resuls displayed in Fig. 6 show another interesting effect, that is relatively narrow distribution of the enhancement factors, which indicates high uniformity of the sample. We have carried out such analysis for several other single nanowires spaced from the corrole molecules by $30 \mathrm{~nm}$ of $\mathrm{SiO}_{2}$ and the result was comparable.

Finally we would like to comment on the possible origin of the observed enhancement. The net effect measured in the experiment is a superposition of enhancement of the absorption and emission rates as well as the fluorescence quenching caused by the nonradiative energy transfer from the molecule to the metallic nanoparticle. Taking into account the spectral properties of corroles and silver nanowires and the fact that a $30 \mathrm{~nm}$ thick $\mathrm{SiO}_{2}$ spacer effectively inhibits the influence of the nonradiative energy transfer, we conclude that the dominant mechanism of the fluorescence enhancement is related to the increase of the absorption rate in the spectral range of plasmon resonance in the metallic nanoparticles.

\section{Summary}

The results of microscopy imaging show clearly that the fluorescence of corrole molecules placed in the vicinity of single silver nanowires can be enhanced due to plasmon induced increase of the absorption rate. The ability to image first the nanowires and then the emission of corroles allows direct and straightforward correlation between nanowire location and the region of enhanced fluorescence. Upon the plasmon enhancement of the fluorescence the emission energy and shape remain unchanged. These observations can be important for improving light-harvesting efficiency in corrole-based artificial molecular assemblies.

\section{Acknowledgments}

Financial support from the WELCOME program "Hybrid nanostructures as a stepping-stone towards efficient artificial photosynthesis" awarded by the Foundation for Polish Science and BOLDCATS project from the European Science Foundation is gratefully acknowledged.
This work was funded by the Foundation for Polish Science (TEAM-2009-4/3). Part of sample characterization measurements was carried out at the facilities of National Laboratory FAMO in the Institute of Physics, NCU in Toruń.

\section{References}

[1] J.R. Lakowicz, Principles of Fluorescence Spectroscopy, Springer, New York 2006.

[2] Th. Basché, W.E. Moerner, M. Orrit, U.P. Wild, Single Molecule Optical Detection, Imaging and Spectroscopy, VCH, Weinheim 1997.

[3] K.P. Hewaparakrama, A. Wilson, S. Mackowski, H.E. Jackson, L.M. Smith, G. Karczewski, Appl. Phys. Lett. 85, 5463 (2004).

[4] K. Karrai, X. Lorenz, L. Novotny, Appl. Phys. Lett. 77, 3459 (2000).

[5] B. Krajnik, T. Schulte, D. Piątkowski, N. Czechowski, E. Hofmann, S. Mackowski, Cent. Eur. J. Phys. 2, 293 (2011).

[6] A. Hartschuh, H.N. Pedrosa, L. Novotny, T.D. Krauss, Science 301, 1354 (2003).

[7] Y. Fu, J.R. Lakowicz, Plasmonics 2, 1 (2007).

[8] P. Anger, P. Bharadwaj, L. Novotny, Phys. Rev. Lett. 96, 113002 (2006).

[9] S. Kühn, U. Håkanson, L. Rogobete, V. Sandoghdar, Phys. Rev. Lett. 97, 017402 (2006).

[10] Ł. Bujak, N. Czechowski, D. Piatkowski, R. Litvin, S. Mackowski, T.H.P. Brotosudarmo, R.J. Cogdell, S. Pichler, W. Heiss, Appl. Phys. Lett. 99, 173701 (2011).

[11] N. Czechowski, P. Nyga, M.K. Schmidt, T.H.P. Brotosudarmo, H. Scheer, D. Piatkowski, S. Mackowski, Plasmonics 7, 115 (2012).

[12] J.B. Nieder, R. Bittl, M. Brecht, Angew. Chem. Int. Ed. 49, 10217 (2010).

[13] S.R. Beyer, S. Ullrich, S. Kudera, A.T. Gardiner, R.J. Cogdell, J. Köhler, Nano Lett. 11, 4897 (2011).

[14] Z. Gross, N. Galili, I. Saltsman, Angew. Chem. Int. Ed. 38, 1427 (1999); R. Paolesse, S. Nardis, F. Sagone, R.M. Khoury, J. Org. Chem. 66, 550 (2001); B. Koszarna, D.T. Gryko, J. Org. Chem. 71, 3707 (2006).

[15] M. Tasior, D.T. Gryko, D.J. Pielacińska, A. Zanell, L. Flamigni, Chem. Asian J. 1, 130 (2010).

[16] D.T. Gryko, B. Koszarna, Org. Biomol. Chem. 1, 350 (2003).

[17] Y. Sun, Y. Yin, B.T. Mayers, T. Herricks, Y. Xia, Chem. Mater. 14, 4736 (2002).

[18] B. Ventura, A. Degli Esposti, B. Koszarna, D.T. Gryko, L. Flamigni, New J. Chem. 29, 1559 (2005). 